13

\title{
Генерация ультракоротких импульсов субтерагерцевого и терагерцевого диапазонов на основе эффекта циклотронного сверхизлучения
}

\author{
(C) Р.М. Розенталь ${ }^{1}$, Н.С. Гинзбург ${ }^{1, \uparrow}$, И.В. Зотова ${ }^{1}$, \\ А.С. Сергеев ${ }^{1}$, В.П. Тараканов ${ }^{2,3}$ \\ ${ }^{1}$ Институт прикладной фризики РАН, Нижний Новгород \\ ${ }^{2}$ Национальный исследовательский ядерный университет „МИФИ“, \\ Москва \\ ${ }^{3}$ Объединенный институт высоких температур РАН, Москва \\ ฯ E-mail: ginzburg@appl.sci-nnov.ru
}

Поступило в Редакцию 18 мая 2017 г.

В рамках усредненного описания электронно-волнового взаимодействия, а также на основе прямого моделирования PIC (particle in cell) показана возможность генерации ультракоротких импульсов субтерагерцевого и терагерцевого диапазонов с использованием циклотронного механизма сверхизлучения (СИ) сгустков электронов, движущихся по винтовым траекториям в однородном магнитном поле. В условиях существенной сверхразмерности волноводного тракта селективное возбуждение рабочей моды обеспечивается при движении электронного сгустка с поступательной скоростью, близкой к групповой скорости излучаемого импульса СИ.

DOI: $10.21883 /$ PJTF.2017.18.45030.16878

В последние годы достигнут существенный прогресс в разработке короткоимпульсных источников на основе эффектов сверхизлучения (СИ) протяженных электронных сгустков. В сантиметровом диапазоне и длинноволновой части миллиметрового диапазона созданы генераторы импульсов субнаносекундной длительности с мультигигаваттным уровнем пиковой мощности, в которых используется черенковский механизм СИ, реализующийся при прямолинейном движении протяженных электронных сгустков в периодических замедляющих системaх [1-3]. Дальнейшее развитие этих исследований предполагает продвижение источников СИ в субтерагерцевый и терагерцевый диапазоны. 
Для достижения этой цели наряду с черенковским механизмом СИ, связанным с возбуждением поверхностных волн в сверхразмерных гофрированных волноводах [4], привлекательно использовать альтернативные механизмы и прежде всего эффект циклотронного СИ. Такое излучение реализуется в регулярном волноводе, когда электроны, составляющие сгусток, двигаются по винтовым траекториям в однородном магнитном поле.

Впервые эффект циклотронного СИ экспериментально наблюдался в диапазоне $38 \mathrm{GHz}$ при значениях ведущего магнитного поля $\sim 1 \mathrm{~T}$ [5]. В соответствии с результатами теоретического анализа [6] максимальная мощность импульсов СИ достигалась в режиме группового синхронизма, когда групповая скорость волны была близка к поступательной скорости движения электронов. В сопровождающей системе отсчета это эквивалентно излучению на квазикритической частоте возбуждаемой рабочей моды, где реализуется режим взаимодействия, аналогичный имеющему место в гиротронах [7]. В таких условиях значительно снижается чувствительность процесса генерации к разбросу скоростей, а также к продольной динамике сгустка. Дополнительным преимуществом режима группового синхронизма является возможность селективного возбуждения рабочей моды в условиях существенной сверхразмерности пространства взаимодействия. В коротковолновых диапазонах необходимость использования сверхразмерных волноводов следует как из условий транспортировки пучков, так и из необходимости снижения до приемлемого уровня пристеночных омических потерь.

Настоящая работа посвящена исследованию возможности генерации импульсов циклотронного СИ в субтерагерцевом и терагерцевом диапазонах при движении электронных сгустков в сильных $(8-10 \mathrm{~T})$ магнитных полях. Условия достижения максимальной пиковой мощности импульсов СИ в режиме группового синхронизма с рабочей модой исследованы в рамках усредненной модели, в которой для описания распространения излучения используется параболическое уравнение. Возможность селективного возбуждения указанной моды в условиях существенной сверхразмерности демонстрируется на основе прямого моделирования PIC (particle in cell) в рамках кода KARAT $[8,9]$.

Будем полагать, что пространство взаимодействия представляет собой отрезок цилиндрического волновода, в котором мода $\mathrm{TE}_{m n}$ возбуждается в режиме группового синхронизма сгустком электронов, вращающихся в однородном магнитном поле $\mathbf{H}=H_{0} \mathbf{z}_{0}$. Представим

Письма в ЖТФ, 2017, том 43, вып. 18 
поперечные компоненты полей рабочей моды в виде

$$
\begin{gathered}
\mathbf{E}_{\perp}=\kappa^{-1} \operatorname{Re}\left(A(z, t)\left[\nabla_{\perp} \Psi \mathbf{z}_{0}\right] e^{i \omega_{c} t}\right), \\
\mathbf{H}_{\perp}=\kappa^{-2} \operatorname{Re}\left(i \partial A(z, t) / \partial z \nabla_{\perp} \Psi e^{i \omega_{c} t}\right),
\end{gathered}
$$

где $A(z, t)$ - медленно меняющаяся комплексная амплитуда поля, $\Psi(r, \varphi)=J_{m}(\kappa r) e^{-i m \varphi}-$ мембранная функция, $\varphi-$ азимутальный угол, $\kappa=\omega_{c} / c, \omega_{c}-$ критическая частота. Процесс циклотронного СИ может быть описан самосогласованной системой уравнений

$$
\begin{gathered}
i \frac{\partial^{2} a}{\partial Z^{2}}+\frac{\partial a}{\partial \tau}=\frac{i G F\left(\tau-g_{0}^{2} Z / 4\right)}{2 \pi} \int_{0}^{2 \pi} \frac{\hat{p}_{\perp}}{\hat{p}_{\|}} d \theta_{0} \\
{\left[\frac{\partial}{\partial Z}+\frac{g_{0}^{2}}{4} \frac{\partial}{\partial \tau}\right] \hat{p}_{\perp}+i \frac{\hat{p}_{\perp}}{p_{\|}}\left(\Delta-1+\left|\hat{p}_{\perp}\right|^{2}+\frac{\hat{p}_{\|}^{2}-1}{g_{0}^{2}}\right)=i \frac{a}{\hat{p}_{\|}}+\frac{\beta_{\perp 0}^{2}}{2} \frac{\partial a}{\partial Z},} \\
{\left[\frac{\partial}{\partial Z}+\frac{g_{0}^{2}}{4} \frac{\partial}{\partial \tau}\right] \hat{p}_{\|}=-g_{0}^{2} \frac{\beta_{\perp 0}^{2}}{2} \operatorname{Re}\left(\frac{\partial a}{\partial Z} \frac{\hat{p}_{\perp}^{*}}{\hat{p}_{\|}}\right),}
\end{gathered}
$$

где использованы нормированные величины:

$$
\begin{gathered}
\tau=\omega_{c} \beta_{\perp 0}^{4} t / 8 \beta_{\| 0}^{2}, \quad Z=\beta_{\perp 0}^{2} \kappa z / 2 \beta_{\| 0}, \quad a=\frac{e A J_{m-1}\left(\kappa R_{0}\right)}{m c \omega_{c} \beta_{\perp 0}^{3} \gamma_{0}}, \\
G=16 \frac{e I_{b}}{m c^{3}} \frac{\beta_{\| 0}}{\beta_{\perp 0}^{6} \gamma_{0}} \frac{J_{m-1}^{2}\left(\kappa R_{0}\right)}{\left(\nu_{n}^{2}-m^{2}\right) J_{m}^{2}\left(v_{n}\right)} ; \\
\hat{p}_{\perp}=\frac{\left(p_{x}+i p_{y}\right) e^{-i \omega_{c} t+i(m-1) \varphi}}{m V_{\perp 0} \gamma_{0}}, \\
\hat{p}_{\|}=p_{\|} / m V_{\| 0} \gamma_{0}
\end{gathered}
$$

- нормированные поперечный и продольный импульсы частиц; $I_{b}$ электронный ток; $J_{m}-$ функция Бесселя; $v_{n}-n$-й корень уравнения $J_{m}^{\prime}(v)=0 ; R_{0}-$ радиус инжекции электронов; $V_{\perp 0}=$ $=\beta_{\perp 0} c \quad$ и $V_{\| 0}=\beta_{\| 0} c-$ начальные значения поперечной и продольной скорости частиц; $g_{0}=\beta_{\perp 0} / \beta_{\| 0}-$ начальный питч-фактор, $\Delta=2\left(\omega_{c}-\omega_{H}\right) / \omega_{c} \beta_{\perp 0}^{2}-$ параметр расстройки между критической частотой рабочей моды и невозмущенным значением гирочастоты 


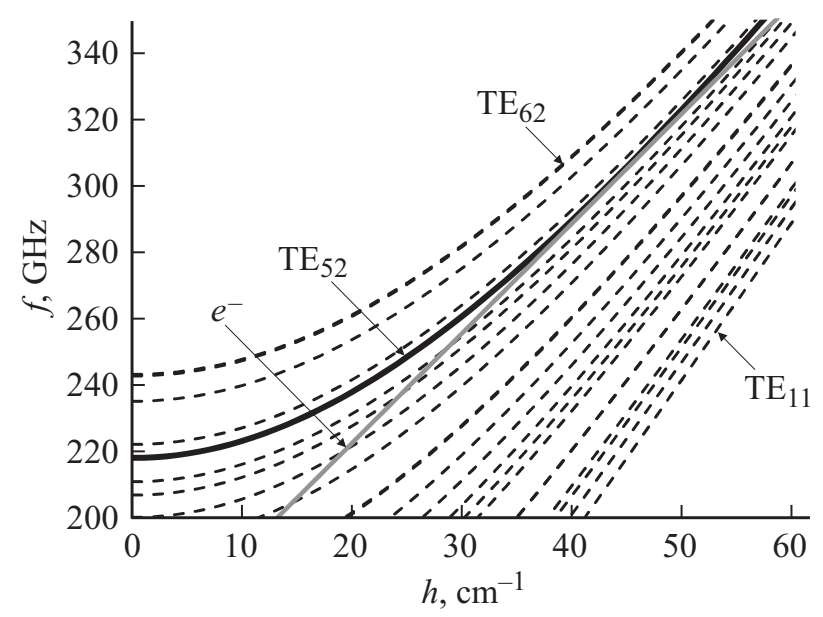

Рис. 1. Дисперсионные кривые электронного пучка $\left(e^{-}\right)$и ТЕ-мод цилиндрического волновода диаметром $5 \mathrm{~mm}$ в окрестности рабочей частоты.

$\omega_{H}=e H_{0} / m c \gamma_{0} ; F-$ функция, описывающая профиль электронного сгустка с длительностью $\Delta \tau$. Будем считать, что на входе в пространство взаимодействия электроны равномерно распределены по фазам циклотронного вращения $p(Z=0)=e^{i \theta_{0}}, \theta_{0} \in[0,2 \pi]$. При этом во входном и выходном сечениях волновода использовались излучательные граничные условия [10].

Отметим, что в отличие от ранее использованных моделей $[5,6]$, где анализ проводился в сопровождающей системе отсчета, уравнения (1)-(3) описывают процессы циклотронного СИ в лабораторной системе отсчета. Возможность анализа непосредственно в лабораторной системе достигается за счет учета временно́й производной $\partial \hat{p}_{\perp, \|} / \partial \tau$ в левых частях уравнений движения [11], что позволяет корректно описать конечность скорости поступательного движения сгустка и представить условия группового синхронизма с рабочей модой в виде $\Delta_{\text {graz }}=1 / g_{0}^{2}$.

В моделировании были использованы параметры электронных сгустков, которые могут быть сформированы на основе сильноточного ускорителя RADAN [12]: энергия частиц $250 \mathrm{keV}$, ток $0.4 \mathrm{kA}$, длительность сгустка 300 ps. Предполагается, что сгусток вращающихся электронов

Письма в ЖТФ, 2017, том 43, вып. 18 

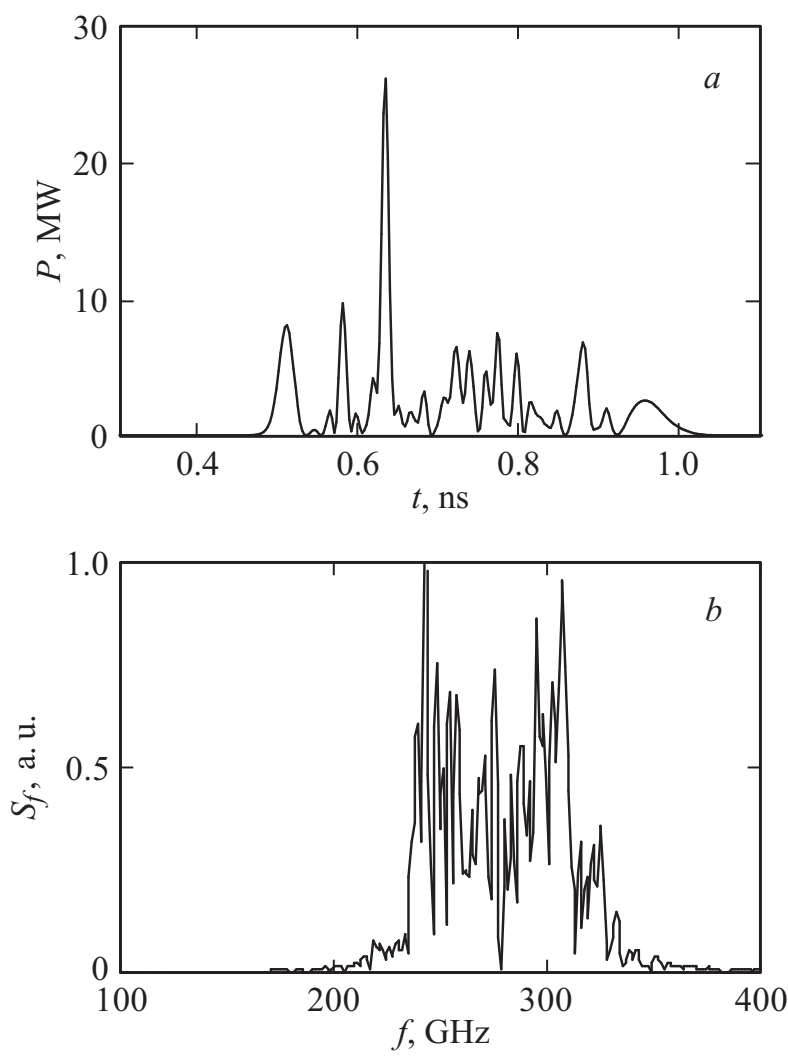

Рис. 2. Моделирование генерации импульсов циклотронного СИ на основе усредненных уравнений: $a-$ зависимость от времени потока мощности на выходе пространства взаимодействия, $b-$ спектр излучения $(L=16, G=35$, $\left.\Delta=11, g=0.3, \beta_{\perp 0}=0.21, \Delta \tau=0.3\right)$.

с питч-фактором $g_{0}=0.3$ движется в цилиндрическом волноводе с длиной $10-15 \mathrm{~cm}$ и диаметром $\sim 5 \mathrm{~mm}$. В этих условиях при значении ведущего магнитного поля $8 \mathrm{~T}$ имеет место режим группового синхронизма с рабочей волной $\mathrm{TE}_{52}$ вблизи частоты $300 \mathrm{GHz}$ (рис. 1).

Моделирование на основе уравнений (1)-(3) показывает, что при длительности электронного сгустка, формируемого ускорителем

Письма в ЖТФ, 2017, том 43, вып. 18 
RADAN, излучение будет состоять из последовательности нескольких импульсов СИ. При этом типичным является выделение одиночного электромагнитного импульса высокой амплитуды. При выбранных параметрах мощность подобного импульса достигает $25 \mathrm{MW}$ при длительности $10 \mathrm{ps}$ в полосе частот $240-310 \mathrm{GHz}$ (рис. 2). Важно отметить, что в энергию излучения в рассматриваемых условиях преобразуется энергия как вращательного, так и поступательного движения, поскольку за счет наличия поперечного магнитного поля возникает продольная компонента силы Лоренца, тормозящая электроны в продольном направлении. Это делает принципиальным учет изменения продольного импульса в уравнениях движения (2), (3). Заметим также, что при уменьшении длительности электронного сгустка до $30 \mathrm{ps}$ реализуется режим генерации одиночных импульсов циклотронного СИ, который был исследован в [6] при анализе указанных процессов в диапазоне $38 \mathrm{GHz}$.

Результаты моделирования на основе усредненной модели подтверждаются расчетами с использованием трехмерной версии РІС-кода KARAT. Для раскачки поперечных осцилляций использовалось сильно неоднородное поле кикера, представляющего собой короткую магнитную катушку, помещенную в поле основного соленоида. Проведенное моделирование с учетом конечного разброса электронного пучка по питч-углам показало прежде всего возможность селективного возбуждения различных мод. Как видно из рис. 3 , по мере изменения значения ведущего магнитного поля последовательно возбуждаются моды $\mathrm{TE}_{42}$ и $\mathrm{TE}_{52}$, для которых имеет место касание (групповой синхронизм) дисперсионных характеристик моды и электронного пучка. Поскольку в сопровождающей системе отсчета, движущейся с поступательной скоростью сгустка, возбуждение рабочей моды происходит на квазикритической частоте [5], подобный механизм селекции аналогичен имеющему место в гиротронах [7]. Максимальная мощность импульсов СИ в режиме группового синхронизма с модой $\mathrm{TE}_{52}$ достигала $3.3 \mathrm{MW}$ при значении магнитного поля $7.9 \mathrm{~T}$ (рис. 3). Омические потери для волновода из меди не превышали $1 \%$. Характерная длительность импульсов СИ составляла $50 \mathrm{ps}$ в полосе частот $240-290 \mathrm{GHz}$.

Отметим в заключение, что дальнейшее радикальное повышение частоты импульсов циклотронного СИ может быть достигнуто при использовании высокоэнергетических электронных сгустков, формируемых на основе фотоинжекторов $[13,14]$. Согласно результатам PIC-моделирования для сгустков с энергией частиц $2 \mathrm{MeV}$, током $200 \mathrm{~A}$ и

Письма в ЖТФ, 2017, том 43, вып. 18 

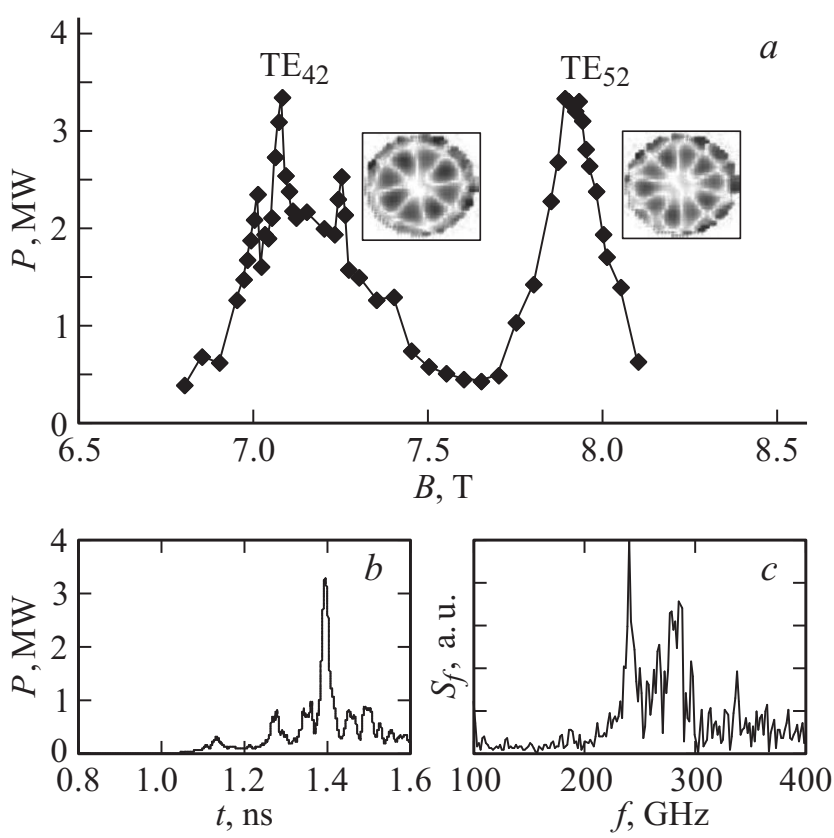

Рис. 3. Результаты трехмерного РІС-моделирования: $a-$ зависимость пиковой мощности импульсов СИ от значения магнитного поля, $b$ и $c-$ соответственно форма импульса СИ и спектр излучения в режиме группового синхронизма с модой $\mathrm{TE}_{52}$.

длительностью $10 \mathrm{ps}$ при движении в ведущем магнитном поле $10 \mathrm{~T}$ в волноводе длиной $35 \mathrm{~cm}$ могут генерироваться импульсы СИ с центральной частотой $\sim 1 \mathrm{THz}$ и мощностью около $10 \mathrm{MW}$ на моде $\mathrm{TE}_{13}$. С учетом того, что в энергию излучения преобразуется энергия как поперечного, так и продольного движения, необходимое значение питч-фактора составляет $g_{0} \approx 0.1-0.15$. Для генерации в коротковолновых диапазонах важное практическое значение имеет существенное снижение величин ведущего магнитного поля в условиях группового синхронизма (по сравнению с используемыми в гиротронах), поскольку при релятивистских поступательных скоростях электронов частота излучения в $\gamma_{\| 0}^{2}$ раз превышает частоту их циклотронных осцилляций. 
Работа выполнена при поддержке Российского научного фонда (грант № 16-42-01078).

\section{Список литературы}

[1] Korovin S.D., Eltchaninov A.A., Rostov V.V. et al // Phys. Rev. E. 2006. Iss. 1. V. 4. P. 016501.

[2] Коровин С.Д., Месяи, Г.А., Ростов В.В. и др. // Письма в ЖТФ. 2004. Т. 30. B. 3. C. $68-74$.

[3] Яландин М.И., Любутин С.К., Рукин С.Н. и др. // Письма в ЖТФ. 2001. T. 27. B. 1. C. $81-88$.

[4] Ginzburg N.S., Malkin A.M., Sergeev A.S. et al // Phys. Rev. Lett. 2016. V. 117. Iss. 20. P. 204801.

[5] Гинзбург Н.С., Зотова И.В., Коноплев И.В. и др. // Письма в ЖЭТФ. 1996. T. 63. B. 5. C. $322-325$.

[6] Гинзбург Н.С., Зотова И.В., Сергеев А.С. // Письма в ЖЭТФ. 1994. Т. 60. B. 7. C. $501-505$.

[7] Petelin M.I. // Plasma Phys. Control. Fusion. 1993. V. 35. Suppl. B. P. B343B351.

[8] V.P. Tarakanov. User's manual for code KARAT. Springfield: BRA, 1992.

[9] Тараканов В.П. // Математическое моделирование. Проблемы и результаты. М.: Наука, 2003. С. 456-476.

[10] Ginzburg N.S., Nusinovich G.S., Zavolsky N.A. // Int. J. Electron. 1986. V. 61. Iss. 6. P. 881-894.

[11] Ginzburg N.S., Sergeev A.S., Zotova I.V., Zheleznov I.V. // Phys. Plasmas. 2015. V. 22. Iss. 1. P. 013112.

[12] Mesyats G.A., Korovin S.D., Rostov V.V. et al // Proc. IEEE. 2004. V. 92. Iss. 7. P. $1166-1179$.

[13] Power J.G. // Proc. of 14th Adv. Accel. Concepts Workshop / Eds G. Nusinovich, S. Gold. Annapolis, 2010. AIP Conf. Ser. 2010. V. 1299. Iss. 1. P. 20-28.

[14] Piot P., Sun Y., Kim K. // Phys. Rev. ST Accel Beams. 2006. V. 9. Iss. 3. P. 031001.

Письма в ЖТФ, 2017, том 43, вып. 18 